\title{
CALCULATION AND REGISTRATION OF THE SAME MOTOR UNIT ACTION POTENTIAL ${ }^{1}$
}

\author{
P.A.M. GRIEP, F.L.H. GIELEN, H.B.K. BOOM, K.L. BOON, L.L.W. HOOGSTRATEN, C.W. POOL and \\ W. WALLINGA-DE JONGE \\ Bio-Information Group, Twente University of Technology, P.O. 217, 7500 AE Enschede, and \\ Zoological Laboratory, University of Amsterdam, Amsterdam (The Netherlands)
}

(Accepted for publication: December 14, 1981)

The electromyogram (EMG) is considered as the summation of a number of motor unit action potentials (MUAPs). Examination of individual MUAPs in order to find characteristics such as shape, duration and amplitude is of appreciable electrophysiological and clinical interest (Hopf and Struppler 1974). For the explanation of the MUAP characteristics it is essential to have insight into the relations between these characteristics and the electrical processes associated with the muscle fibre membranes, together with anatomical and physiological data of a motor unit (MU).

In the past much effort has been directed to gaining such insights with the aid of more and more sophisticated measuring methods. For this coaxial, multi and single fibre and micro/macro-needle electrodes have been used (Buchthal et al. 1957; Ekstedt 1964; Stålberg and Trontelj 1979). However, precise interpretation of the obtained signals is difficult because no adequate theory of the origin and volume conduction of the EMG phenomena is available.

Significant theoretical work concerning volume conduction was done by Lorente de Nó (1947), Clark and Plonsey (1966), Rosenfalck (1969), Dimitrova (1974) and Plonsey (1974), who related field potentials to bio-

\footnotetext{
1 This work was supported by a grant from The Netherlands Organization for the Advancement of Pure Research (ZWO).
}

electrical sources. Knowledge on motor unit structure was considerably expanded by the introduction of histochemical techniques, viz., staining of the motor endplates (Coërs and Woolf 1957) and selective staining of the fibres of one motor unit (Kugelberg 1973).

This information is, however, hardly used in the EMG models reported in the literature. If only global structures of motor units are taken into account, it is difficult to interpret recorded MUAPs. Some models are based on merely statistical descriptions (George 1970; Lindström and Broman 1974; thesis Lindström, Chalmers University of Technology, Göteborg 1974), some are based on specific arrangements of muscle fibres (Boyd et al. 1978; Dimitrov 1978a, b; Griep et al. 1978; Wani and Guha 1980). In our opinion the models which are based on realistic arrangements of muscle fibres are most promising for interpreting MUAP shapes and in order to verify this idea a special experiment was carried out.

In our experiment only one single motor unit was stimulated and the MUAP of this unit was recorded with several intramuscular wire electrodes. After the experiment the positions of the electrodes together with the positions of the activated fibres were found by means of histochemical techniques. Other parameters, like conduction velocity of the muscle fibres and electrical properties of the medium were derived from other experiments 
or the literature. The field potential contribution at the electrode position of each muscle fibre of the motor unit was then calculated. The superposition of these contributions resulted in the calculated MUAP, which was compared with the measured MUAP. The chief points emerging from this approach are discussed.

\section{Calculation of the MUAP}

A motor unit is supposed to consist of straight cylindrical muscle fibres which run parallel to each other.

The geometry of an arbitrary muscle fibre $i$ is then characterized by the geometrical parameters $x_{i}, y_{i}, d_{i}, z_{1 i}, z_{2 i}, z_{3 i}$ (see Fig. 1). The activation time of each fibre of a motor unit is denoted by $t_{i}$ : the time of activation of fibre $i$ at the endplate. In order to calculate MUAPs the following starting points are used:

(a) The extracellular potential (the MUAP) consists of linearly summated single fibre potentials $\phi_{\mathrm{i}}$ from all fibres of the motor unit.

(b) The extracellular medium behaves as a purely resistive, homogeneous and infinite medium with cylindrical anisotropy. This implies that the medium is characterized by two conductivities $\sigma_{\mathrm{r}}$ and $\sigma_{\mathrm{z}}$ in the radial and longitudinal direction of the fibres respectively.

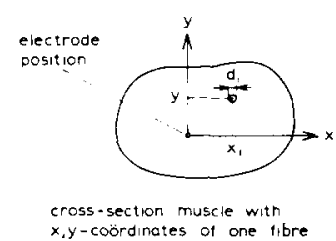

(A)

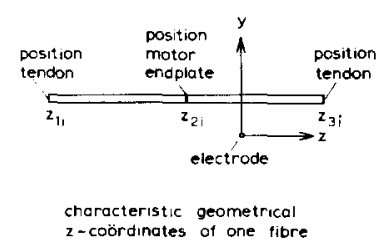

(B)
Fig. 1. Definition of the geometry parameters of a muscle fibre. The origin of a cartesian coordinate system coincides with the position of the electrode. A: cross-section of the whole muscle, $x_{i}, y_{i}=$ coordinates of fibre $i$ with respect to the position of the electrode; $d_{i}=$ diameter of fibre $i$. The distance of fibre $i$ to the electrode is $r_{i}=\left(x_{i}^{2}+y_{i}^{2}\right)^{1 / 2} . B$ : longitudinal section of fibre $i . z_{1 i}, z_{2 i}, z_{3 i}$ indicate respectively the $z$ position of one end of the fibre, the position of the motor endplate and the other end of the fibre. (c) In an isotropic extracellular medium $\left(\sigma_{\mathrm{r}}=\sigma_{\mathrm{z}}=\sigma_{\mathrm{e}}\right)$ the single fibre potential (SFP) $\phi^{\prime}$ at the electrode position is descibed by:

$\phi^{\prime}(r, t)=\frac{1}{4 \pi \sigma_{e}} \int_{-\infty}^{\infty} \frac{i_{m}(z)}{\left(r^{2}+z^{2}\right)^{1 / 2}} d z$

(Rosenfalck 1969)

with:

$\mathrm{i}_{\mathrm{m}}(\mathrm{z})=\frac{\sigma_{\mathrm{in}} \cdot \pi \cdot \mathrm{d}^{2}}{4}\left(\frac{\mathrm{d}^{2} \mathrm{~V}_{\mathrm{in}}(\mathrm{z})}{\mathrm{dz}^{2}}\right)$

where $i_{m}(z)=$ transmembrane current per length unit as function of $z$ and $z=z(t)$; $\mathrm{V}_{\text {in }}(\mathrm{z})=$ intracellular potential as function of $\mathrm{z} ; \sigma_{\text {in }}=$ intracellular conductivity; $\mathrm{d}=$ diameter of fibre $\mathrm{i} ; \sigma_{\mathrm{e}}=$ extracellular conductivity; $z=$ distance in $z$-direction between source $i_{m}(z)$ and electrode $(z=0) ; r=\left(x_{i}^{\tilde{2}}+y_{i}^{2}\right)^{1 / 2}$ distance from electrode to fibre $i$. Notice that the current $i_{m}(z)$ is determined by the second derivative of the intracellular potential $V_{\text {in }}(z)$ ! The potential $\phi$ for a cylindrically anisotropic medium $\left(\sigma_{\mathrm{r}} \neq \sigma_{\mathrm{z}}\right)$ can be obtained from eq. (1) by a transformation:

$$
\begin{aligned}
& \phi(\mathrm{r}, \mathrm{t})=\phi^{\prime}(\mathrm{Kr}, \mathrm{t})= \\
& \quad=\frac{1}{4 \pi \sigma_{e}} \int_{-\infty}^{\infty} \frac{\mathrm{i}_{\mathrm{m}}(\mathrm{z})}{\left(\mathrm{K}^{2} \mathrm{r}^{2}+\mathrm{z}^{2}\right)^{1 / 2}} \mathrm{dz}
\end{aligned}
$$

(Plonsey 1974)

$$
\begin{aligned}
& \text { with } \mathrm{K}=\left(\frac{\sigma_{\mathrm{z}}}{\sigma_{\mathrm{r}}}\right)^{1 / 2} \\
& \text { and } \sigma_{\mathrm{e}}=\sigma_{\mathrm{r}}
\end{aligned}
$$

(d) The depolarization is generated at the motor endplate and travels to both fibre ends. According to Rosenfalck (1969) the extracellular potential can be calculated accurately if the intracellular action potential $V_{i}$ is approximated by a triangular shape. In this approach (see Fig. 2) the single fibre potential $\phi$ may be considered to be caused by a set of 3-point current sources:

$$
\phi(r, t)=\frac{1}{4 \pi \sigma_{r}} \sum_{p=1}^{3} \frac{I_{p}}{\left(K^{2} r^{2}+z_{p}^{2}\right)^{1 / 2}}
$$



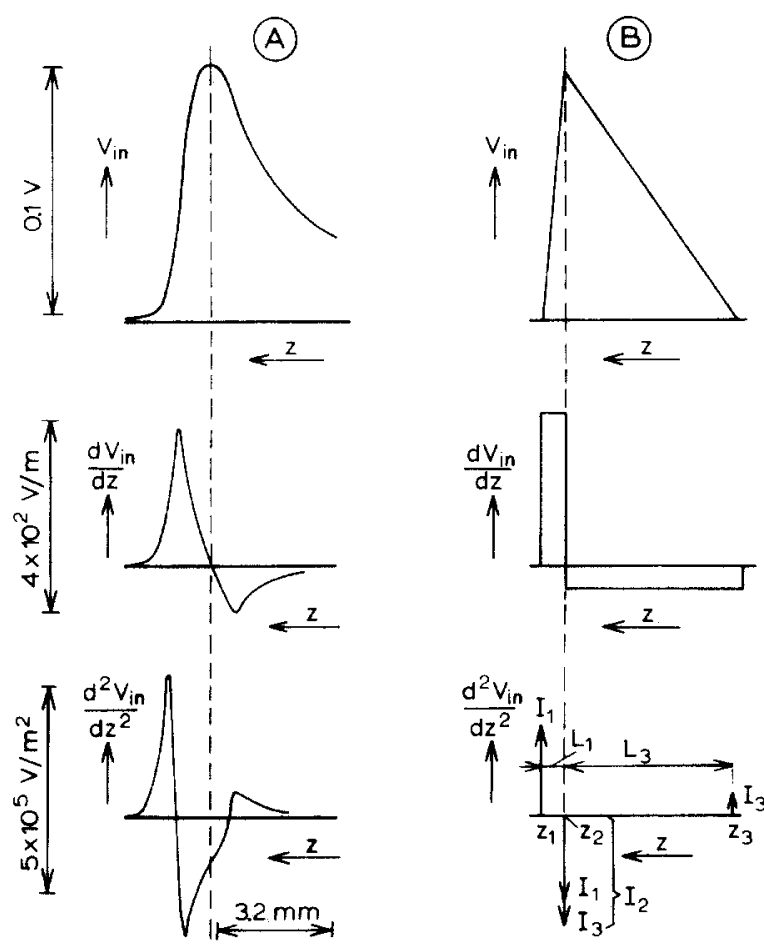

Fig. 2. Introduction of the tripole model description of the electrical phenomena of an active muscle fibre. A: a picture of the intracellular action potential $V_{i}(z)$ and its first and second derivatives. The $V_{i}(z)$ represents the intracellular action potential during the propagation phase of an EDL muscle fibre of the rat (derived from Hanson 1974) as a function of the longitudinal position $\mathrm{z}$ (the conduction velocity used to transform the results of Hanson from the time to the distance domain is $3.2 \mathrm{~m} / \mathrm{sec}$ ). B: a schematic representation of the intracellular action potential and its first and second derivatives as they are related to the tripole model of Rosenfalck (1969). The tripole, shown in the bottom picture, can be considered as a combination of two dipoles.

where: $I_{p}=$ strength of point current source $p$; $z_{p}=$ position of point current source $p$. Notice that the position of each point current source will change in $\Delta t$ seconds with $v \cdot \Delta t$ where $\mathrm{v}$ represents the conduction velocity of the action potential; so $z_{p}=z_{p}(v \cdot \Delta t)$.

(e) According to assumptions in the volume conduction theory the medium is considered as resistive (no charge will be accumulated). Then the total current through the fibre mem-

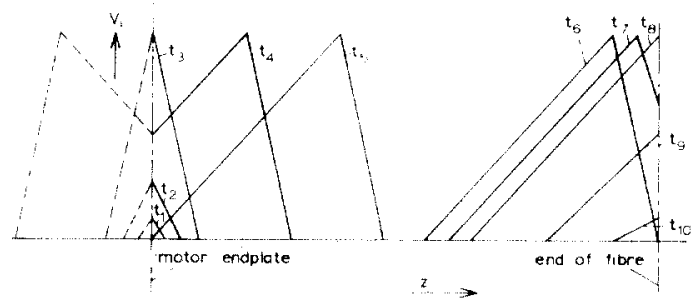

Fig. 3. Intracellular potentials according to the tripole model at successive points in time during the 3 phases viz. generation $\left(t_{1}, t_{2}, t_{3}, t_{4}\right)$, propagation $\left(t_{5}, t_{6}\right)$ and extinction $\left(t_{7}, t_{8}, t_{9}, t_{10}\right)$.

brane is zero at any time:

$I_{1}+I_{2}+I_{3}=0$

(f) As a consequence of the fact that the internal potential returns to its resting potential, the sum of the 'dipole strengths' of the current sources is zero:

$\left(I_{1} \times L_{1}\right)+\left(I_{3} \times L_{3}\right)=0$ (Plonsey 1969; Ch. 5.9 , pages 237 and 239 )

where: $\mathrm{L}_{1}=\mathrm{z}_{1}-\mathrm{z}_{2} ; \mathrm{L}_{3}=\mathrm{z}_{3}-\mathrm{z}_{2}\left(\mathrm{z}_{1}, \mathrm{z}_{2}\right.$ and $z_{3}$ indicate the positions of 3 poles, see Fig. 2).

(g) At time $t_{i}$ the action potential is generated at the motor endplate (position $z_{2 i}$ ). In the model this generation is described by means of a linear increase in time of both the current amplitude and the lengths $L_{1}$ and $L_{3}$ in accordance with the formulas 4 and 5. The extinction of the action potential at the end of the fibre is described by means of a linear decrease of current amplitude and lengths $L_{1}$ and $L_{3}$. This approach for the generation and extinction phases has been described in more detail by Griep et al. (1979) and is illustrated in Fig. 3.

(h) The potential contribution of the action potential of fibre $i$ which travels in the opposite direction is calculated similarly and added to the first one.

\section{Which parameters have to be determined?}

In this section only a general survey of the parameters needed to calculate a MUAP is 
given. Below we shall give more details about the actual values which were used.

A MUAP can be calculated by means of the formulas given in the previous section. This means that for each activated fibre the following parameters have to be substituted: the tripole parameters $I_{1}, I_{2}, I_{3}, L_{1}$ and $L_{3}$; the conduction velocity $v$; the activation time $t_{i}$; the position of the motor endplate $\left(z_{2 i}\right)$ and the positions of the ends of the fibre $\left(z_{1 i}\right.$, $\mathbf{z}_{3 \mathrm{i}}$ ); the electrode position and furthermore the distance $r$ of each fibre from the electrode. Besides these parameters the conductivities of the extracellular medium $\sigma_{z}$ and $\sigma_{x}$ and the conductivity of the intracellular medium $\sigma_{i}$ and the fibre diameter have to be known.

It was assumed that the tripole parameters and the conduction velocity were the same for all fibres of the MU. The basis for both assumptions is given in the section on Tripole parameters and conduction velocity. The tripole parameters were derived from the literature. The conduction velocity was determined from our own measurements.

The activation times $t_{i}$ of the muscle fibres show slight random variations; the interval time in which all fibres are activated was estimated to be $0.12 \mathrm{msec}$. The activation moment of each fibre was described with the aid of a probability density function (see below).

The positions $z_{1 i}, z_{2 i}$ and $z_{3 i}$ were derived from anatomical data. As will be shown in Experiments, extensor digitorum longus (EDL) muscles of the rat were used for the measurements. The geometry of a number of EDL muscles was studied and it turned out that the geometrical structure could be represented as a parallelepiped. In this parallelepiped the endplate region was represented by a set of 3 straight planes (see Fig. 6).

The distance $r$ of each fibre to the electrode is very important, as can be concluded from eq. (3). Therefore the coordinates $x_{i}$ and $y_{i}$ with respect to the electrode position (defined in Fig. 1) were determined for each fibre separately by means of histochemical

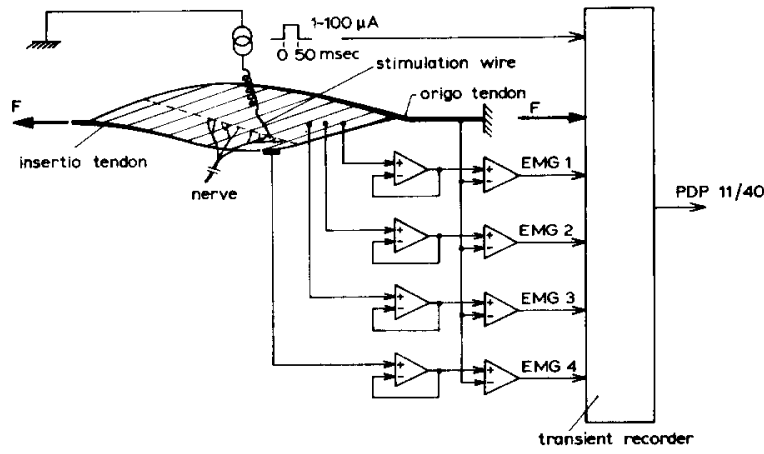

Fig. 4. Schematic drawing of the experimental arrangement. One motor unit is stimulated using the epimuscular stimulation method. The EMG is measured with electrodes in and upon the muscle.

labelling. The diameter of each fibre could also be quantified but was assumed to be the same for all fibres (see below). The position of the electrode(s) in the muscle was found by means of specific histochemical techniques (see Fig. 5). The conductivities $\sigma_{\text {in }}, \sigma_{z}$ and $\sigma_{\mathrm{r}}$ were obtained from the literature (see below).

\section{Experiments}

\section{(1) Muscle preparation}

The extensor digitorum longus muscle (EDL) of the rat was used for our investigations. This muscle contains many motor units with low resistance to fatigue, thus facilitating the labelling of the fibres by means of glycogen depletion.

The EDL of adult male Wistar rats was prepared free (blood flow unimpaired, muscle in moist air at $37^{\circ} \mathrm{C}$ ). The ischiadic nerve was cut at about $3 \mathrm{~cm}$ from the muscle. The origin tendon was fixed and the insertion tendon was cut and attached to an isometric force transducer (passive muscle force was adjusted to $0.02 \mathrm{~N}$ ). A survey of the general design is given in Fig. 4.

\section{(2) Stimulation and MUAP recording}

A single motor unit was stimulated in a stable way by a Pt-Ir wire electrode (diameter 
$25 \mu \mathrm{m})$ positioned on the surface of the muscle. Griep et al. (1980) showed clearly that this method of stimulation (the so-called epimuscular microstimulation) triggers a single motor unit via excitation of an axon terminal.

The MUAP on the surface of the muscle was measured with a small surface electrode, positioned with a micromanipulator. The territory of the motor unit within the muscle was roughly estimated by using this surface electrode. After this estimation intramuscular wire electrodes (Pt-Ir, diameter $25 \mu \mathrm{m}$, teflon isolated except at the $\mathrm{Ag}$ coated end of the wire) were put in the motor unit territory by means of forceps. The surface EMG, the wire EMGs and the force were measured and monitored on a memory oscilloscope.

The input impedance of the EMG amplifiers, including the input wires, was $6 \mathrm{pF} / / 100$ $\mathrm{M} \Omega$ with a bandwidth of $0.5 \mathrm{~Hz}-30 \mathrm{kHz}$ and a slope of $18 \mathrm{~dB} /$ octave. The measured signals were buffered in a transient recorder (each channel: maximum sample frequency 100 $\mathrm{kHz}, 2048 \times 10$ bits) that was connected online with a PDP11/40 computer.

\section{(3) Labelling of the fibres and the electrode position}

Labelling of the fibres of the MU was effected by depletion of their glycogen content by means of continuous stimulation for $3 \mathrm{~min}$ at a frequency of $10 \mathrm{~Hz}$. Just before the end of this stimulation period the positions of the intramuscular wire electrodes were marked in the muscle tissue by Ag-electrophoresis (anodic DC current, $1 \mu \mathrm{A}$ during $15 \mathrm{sec})$. Immediately after the continuous stimulation period the muscle was fixed in a special clamp, excised and immersed in liquid Freon-22 and cooled in liquid nitrogen (the whole procedure was performed within 5 $\min$ ).

\section{Determination of the parameters}

In the following 5 sections, the following parameters will be described: distance of the activated fibres from the electrode; positions of the motor endplates and of the fibre ends of the activated fibres; tripole parameters and conduction velocity of the action potential; electrical conductivities and anisotropy of the extracellular medium; dispersion in activation time.

\section{(1) Distance of the activated fibres from the electrode}

In order to determine the distance of each activated fibre from the electrode, the muscle embedded in paraffin was cut into sections (thickness $6 \mu \mathrm{m}$ ) approximately perpendicular to the fibre direction.

The sections were divided into two groups. The first consisted of the section numbers 1 , $2,5,6,9,10$, etc. They were stained with the PAS (periodic acid Schiff) reaction to identify the glycogen depleted fibres (Pool et al. 1978). In this way the positions of all fibres of the motor unit were found. The second group was stained to make the silver deposits visible (Baumgarten et al. 1960) thus identifying the position(s) of the electrode(s). It turned out that afterwards these positions could also be found in the PAS stained sections.

In photographic enlargements of these sections the distance of each activated fibre (centre of the fibre) to the electrode(s) was measured with an accuracy of $\pm 10 \mu \mathrm{m}$. With respect to the cross-sections of the individual fibres of the activated motor unit (shown in Fig. 5) we found a mean area of $19 \times 10^{2} \mu \mathrm{m}^{2}$ with a standard deviation of $3 \times 10^{2} \mu \mathrm{m}^{2}$. The mean area and the standard deviation of these fibres of the motor unit are representative of the fast fatiguable EDL motor units.

In the formulae instead of an area the fibre diameter was used. In the computer program we introduced a diameter of $48 \mu \mathrm{m}$. This value corresponds with a mean area of $18 \times$ $10^{2} \mu \mathrm{m}^{2}$.

Fig. 5 shows a photograph of a section in which the position of an electrode together with the positions of the fibres of the depleted motor unit are visible. Some white fibres (rather frequently occurring at the cir- 


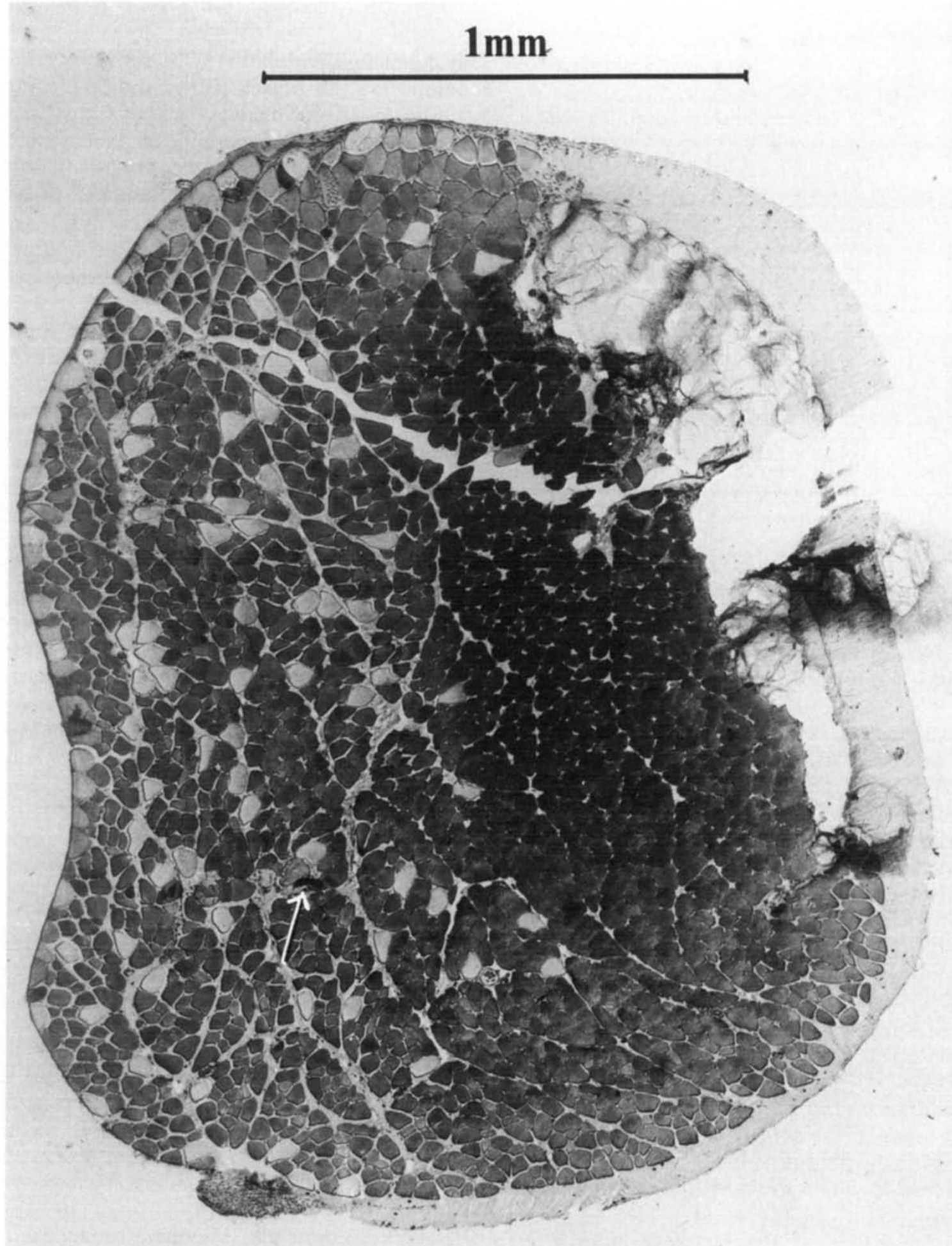

Fig. 5. Photograph of a muscle section (perpendicular to the muscle fibres) in which the position of an electrode (electrode 2) and the positions of the activated fibres are visible (exp. 064). The electrode position is indicated with an arrow, the light coloured fibres belong to the MU investigated. The structure at the upper left part of the cross-section is the tendon of origin. The position of the cross-section is schematically indicated in the right part of Fig. 7C. 


\section{TABLE I}

Information concerning the number of muscle fibres per motor unit for two experiments (exp. 063 and exp. 064) and the position of the nearest and furthest muscle fibres with respect to the electrodes (electrodes 1 and 2 ).

\begin{tabular}{llll}
\hline Exp. & $\begin{array}{l}\text { Fibres/ } \\
\text { MU }\end{array}$ & $\begin{array}{l}\text { Distance } \\
\text { of nearest } \\
\text { fibre }(\mu \mathrm{m})\end{array}$ & $\begin{array}{l}\text { Distance } \\
\text { of furthest } \\
\text { fibre }(\mu \mathrm{m})\end{array}$ \\
\hline $\begin{array}{l}\text { Exp. 063 } \\
\text { electrode 1 }\end{array}$ & 98 & 210 & 1790 \\
$\begin{array}{l}\text { Exp. 063 } \\
\text { electrode 2 }\end{array}$ & 98 & 90 & 2260 \\
$\begin{array}{l}\text { Exp. 064 } \\
\text { electrode 1 }\end{array}$ & 73 & 100 & 1880 \\
$\begin{array}{l}\text { Exp. 064 } \\
\text { electrode 2 }\end{array}$ & 73 & 60 & 1490 \\
\hline
\end{tabular}

cumference of the muscle) were detected as artefacts when using 4 extra muscle crosssections, two on each side of the section in which the electrode was found. These false fibres were eliminated from the MUAP calculation.

According to Griep et al. (1980) only one motor unit was stimulated: the motor unit force decreased smoothly and the MUAP shape changed gradually.

Clear cracks (as can be seen in Fig. 5) are artefacts. In the cross-sections the cracks were taken into account when determining the positions of the motor unit fibres with respect to the EMG electrode. Shrinkage due to the histochemical procedure was estimated to be less than 5\% (Pool, personal communication).

Detailed information derived from muscle cross-sections is given in Table I.

(2) Positions of the motor endplates and of the fibre ends of the activated fibres

In Fig. 6A a photograph of an EDL muscle is presented in which the positions of the endplates are visible. In the cross-section of the muscle the width of the endplate zone appeared to be about $0.3 \mathrm{~mm}$. Fig. $6 \mathrm{~B}$ shows a schematic representation of the muscle, derived from 5 EDL muscles with histochemi- cally identified endplates. With respect to the geometry of the muscle fibres the following assumptions were made: (i) the fibres are straight; (ii) they run parallel to each other; (iii) they all have the same length (Close 1964). The tendon plates were assumed to be flat and also to run parallel to each other.

The coordinates of each fibre and therefore the distance of each fibre from the electrode were measured from photographs (see previous section), but it was hardly possible to find for the same fibre the position of its motor endplate $\left(z_{2 i}\right)$ and the junctions at the tendons $\left(z_{1 i}, z_{3 i}\right)$. These 3 parameters $\left(z_{1 i}, z_{2 i}\right.$, $z_{3 i}$ ) were derived from the geometrical model of Fig. 6B. The general geometry of the muscle was approximated by a parallelepided. The parameters $l_{f}$ and $l_{m}$ of the muscles used were measured from photographs taken during the experiments.

The angle $\alpha$ between the fibres and the tendon plates was calculated in two ways (see Fig. 6B). Firstly it was derived from the muscle thickness $d$ and the fibre length $l_{f}$ according to $\alpha=\arcsin \left(d / l_{f}\right)$. Secondly the angle was determined from $\arctan (\mathrm{CD} / \mathrm{CB})$. For the calculations the mean of the two values was taken (accuracy approximately $\pm 2^{\circ}$ ).

The value $l_{c}$ was obtained experimentally in the following way: a surface electrode was shifted along the line $A B$ to a position where the delay between stimulus pulse and negative EMG onset was minimal.

In Table II the values of the geometrical model have been summarized for the two muscles that were used in the plots of Figs. 7 and 8.

Some special remarks have to be made with respect to the position of the endplates $\left(z_{2 i}\right)$. Notice that the geometrical model represents the endplate zone by means of straight planes with zero thickness. Since this zone has actually a thickness of approximately $0.3 \mathrm{~mm}$, we introduced for the computations of the MUAPs a random variable that was superimposed on the values $z_{2 i}$. This variable was distributed according to a uniform probability density function with a width corresponding 

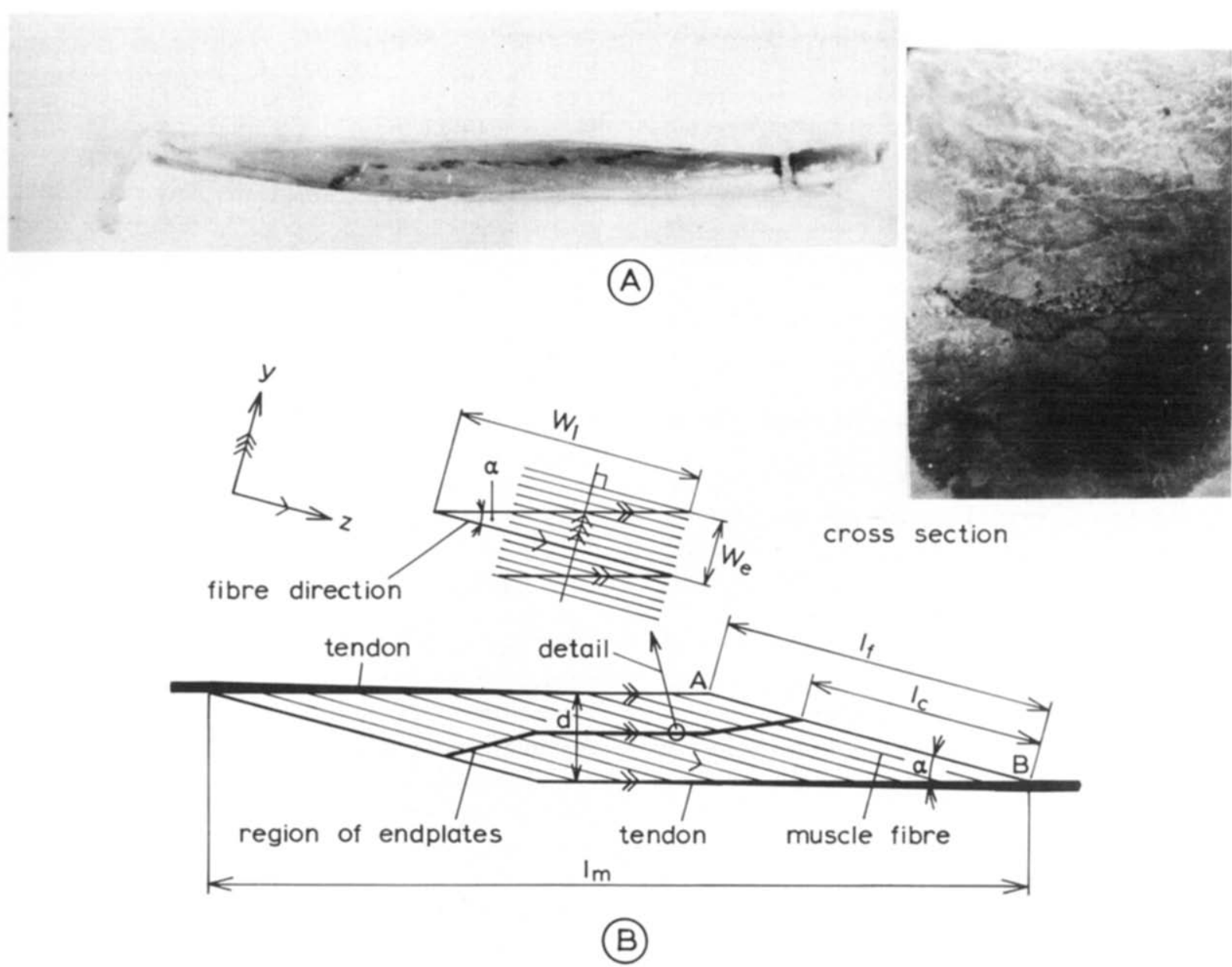

Fig. 6. A: example of an EDL muscle with histochemically (acetylcholinesterase) coloured endplates. The crosssection shown at the right is taken perpendicular to the muscle fibres through the endplate region; notice the positions of the endplates. B: a schematic representation of a side view of an EDL muscle derived from 5 EDL muscles with histochemically identified endplates. A part of the endplate region is shown in more detail. Symbols used are: $l_{f}$, fibre length $; l_{m}$, muscle length; $l_{c}$, distance between tendon and endplate zone, along a muscle fibre at the periphery of the muscle; $\alpha$, angle between muscle fibres and tendon plate; $W_{e}$, width of the endplate zone perpendicular to the muscle fibres; $W_{1}$, width of the endplate zone in the direction of the muscle fibres; $A$, end of tendon plate; $B$, end of muscle fibre tissue.

\section{TABLE II}

Geometrical parameters of two muscles. The parameters have been defined in Fig. 6B.

\begin{tabular}{lcc}
\hline Parameter & Exp. 063 & Exp. 064 \\
\hline $1_{\mathrm{f}}(\mathrm{mm})$ & 15.0 & 15.3 \\
$1_{\mathrm{m}}(\mathrm{mm})$ & 35.9 & 34.4 \\
$1_{\mathrm{c}}(\mathrm{mm})$ & 10.5 & 10.7 \\
$\alpha($ degrees $)$ & 15 & 12 \\
$\mathrm{~W}_{\mathrm{e}}(\mathrm{mm})$ & 0.3 & 0.3 \\
\hline
\end{tabular}

to the actual measured thickness of the motor endplate zone.

(3) Tripole parameters and conduction velocity

Data of intracellular potentials $V_{\text {in }}$ as found in the literature (Muscholl 1957; Yonemura 1967; Hanson 1974; Ludin 1976) were used to obtain the tripole parameters $I_{1}, I_{2}$ and $L_{1}$. In order to deduce these param- 
eters photographic enlargements of $V_{\text {in }}$ registrations were used. The second derivative $\mathrm{d}^{2} \mathrm{~V}_{\mathrm{in}} / \mathrm{dt}^{2}$ of such a registration was calculated numerically. From this second derivative (see also Fig. 2) the tripole parameters were derived, taking into account the conduction velocity of the action potential. The pole strengths $I_{1}$ and $I_{2}$ were taken proportionally to the areas of the first and second phases, respectively. The third pole strength $I_{3}$ followed from the condition $I_{1}+I_{2}+I_{3}=0$. The value $L_{1}$ was equalized to the distance between the first two extremes of the triphasic curve. The value $\mathrm{L}_{3}$ followed from the condition $I_{1} L_{1}+I_{3} L_{3}=0$. Notice that we used length intervals (e.g., $L_{1}$ ) instead of time durations (e.g., duration between poles) on using the transformation $\mathrm{L}=\mathrm{v} \cdot \mathrm{t}$, in which $\mathrm{v}$ represents the conduction velocity.

The conduction velocity was derived from experiments in which a single MUAP was measured at several positions along the surface of the muscle. In this way a conduction velocity of $3.2 \mathrm{~m} / \mathrm{sec}$ was found (temperature $37^{\circ} \mathrm{C}$; accuracy: $0.5 \mathrm{~m} / \mathrm{sec}$ ). The accuracy was influenced by the fact that the shape of the action potential depended on the position along the muscle fibres.

The parameters $I_{1}, I_{2}$ and $L_{1}$, which were actually used for the computations, were compromises of the divergent values derived from the action potentials described in the literature. Finally for $L_{1}$ a value of $450 \mu \mathrm{m}$, for the ratio $I_{1} / I_{2}$ a value of 0.80 and for $I_{2}$ a value of $0.50 \mu \mathrm{A}$ were taken. Because of the large variations in the values derived from the literature it is rather misleading to give values of accuracies with respect to the tripole parameters.

\section{(4) Electrical conductivities and anisotropy}

The electrical conductivities $\sigma_{\mathrm{z}}$ and $\sigma_{\mathrm{r}}$ and therefore the degree of anisotropy $\sigma_{z} / \sigma_{r}$ of the extracellular medium are not precisely known. According to Rush et al. (1963, Table 1) the conductivities $\sigma_{z}$ and $\sigma_{x}$, measured by the so-called 4-point method, lie in the following ranges:
$0.41<\sigma_{\mathrm{z}}<0.80(\Omega \mathrm{m})^{-1}$

$0.04<\sigma_{\mathrm{r}}<0.21(\Omega \mathrm{m})^{-1}$.

For the degree of anisotropy Geddes and Baker (1967) mentioned in their review article a range of $1.8-15.3$.

For our calculations the degree of anisotropy was chosen as 5 and for the conductivities $\sigma_{\mathrm{z}}$ and $\sigma_{\mathrm{r}}$ the values 0.75 and 0.15 $(\Omega \mathrm{m})^{-1}$ were taken.

For the intracellular conductivity $\sigma_{\text {in }}$ a value of $0.4(\Omega \mathrm{m})^{-1}$ was used (Fatt 1964).

Notice that it is the value $\sigma_{z} / \sigma_{\mathrm{r}}$ that affects the normalized shape of a single fibre potential. The absolute values of the conductivities are only important with respect to the accuracy of the amplitude of a MUAP.

\section{(5) Dispersion in activation time}

The time between the moment of stimulation and the moment of activation of the fibres of the motor unit is not the same for each fibre. This time is composed of the time needed for conduction along the nerve twigs and the endplate delay time. For the EDL muscle the mean interval was approximately 0.4 msec (Griep et al. 1980, Fig. 2). According to Hubbard and Schmidt (1963) the endplate delay time for the diaphragm muscle of the rat is approximately $0.22 \mathrm{msec}$.

It was impossible to determine the moment of activation of each fibre separately. The differences in moment of activation with respect to the moment of activation of the first fibre were represented in our model by means of a uniform probability density function with a width of $120 \mu \mathrm{sec}$. This width results from the differences in the conduction times along the twigs of the axon of the motor unit and the differences in the endplate delay times. Both differences are not quantitatively known for the rat EDL motor units. The width of the distribution function was inevitably chosen more or less arbitrarily.

Notice that the interval between the moment of stimulation and the moment of activation of the first fibre is constant and only affects the relative position of the MUAP on the axis but not the shape. 
The shape is dependent on the distribution function of the moment of activation of the fibres of the motor unit. From pilot studies it turned out that the fibre that is closest to the electrode is very important with respect to the shape of the MUAP. If in a registration a sharp peak was observed, then there was a fibre in very close vicinity to the electrode in the photograph of the cross-section. In such a case a special calculation procedure was followed: both the moment of activation and the endplate position of this closest fibre were kept constant. Their values were adjusted in such a way that the sharp peak in the calculated MUAP occurred at the same position in comparison with the total shape of the measured MUAP. The activation moments and endplate positions of the other fibres were randomly selected from the appropriate distribution functions. On applying this procedure several times a bundle of curves is obtained. Such a bundle shows in fact the effect of the variability of the parameters chosen. We called this procedure 'the clamping procedure.' If no remarkable peak in the measured MUAP was found no clamping procedure was performed: the moment of firing of each fibre was taken randomly from the appropriate distribution function.

\section{Comparison between measured and calculated MUAPs}

The measured MUAPs obtained during two experiments, exp. 063 and exp. 064 respectively were used in order to evaluate the MUAP model and therefore our insights with respect to the generation of MUAPs.

Fig. 7A shows the MUAPs of both experiments as measured at two different electrode positions. The peak at time zero represents the stimulus artefact. In Fig. 7B the corresponding calculated MUAPs are shown. Notice that in fact a bundle of curves is given for each situation. MUAP2 of exp. 063 and MUAP2 of exp. 064 are bundles obtained by the clamping procedure (see previous section).
Fig. 7C shows the position of the electrodes in the muscle as it is represented by means of the geometrical model.

At first sight, the resemblance between measured and calculated MUAPs is 'rather good.' Of course we have to realize that the absolute values are strongly dependent on the electrical conductivities, parameters that are in fact poorly known.

The basic shape of the measured MUAPs is always triphasic, with a positive initial phase. We have to keep in mind that the shape actually recorded will strongly depend on the position of the electrode. At the MUAP denoted by MUAP2 exp. 064 a very steep positivenegative slope can be observed. This indicates that the electrode was really close to an active fibre. Inspection of the cross-section of the histochemically coloured muscle confirmed this.

With respect to the degree of similarity between the shapes of actually recorded and calculated MUAPs the following remarks can be made: (a) both the measured and the calculated MUAPs are triphasic; (b) in both experiments MUAP2 is larger than MUAP1; (c) the durations of the calculated and measured MUAPs differ but not in a deterministic way; this means that the calculated durations are sometimes too short and at other times too long; (d) if the amplitude of the first positive phase in the measured MUAP is larger or smaller than the amplitude of the second positive phase, then the amplitude of the first positive phase in the calculated MUAP is also larger or smaller than the amplitude of the second positive phase; (e) MUAP2 (exp. 064) shows a pronounced peak in the negative phase; this peak is apparently related to the single fibre potential of the fibre that was in close vicinity to the electrode; it can also be observed in the bundle of calculated MUAPs; (f) there is a remarkable difference between measured and calculated MUAPs with respect to the manner of increase during the onset phase and decrease during the extinction phase.

In the next section it will be demonstrated 
(A)

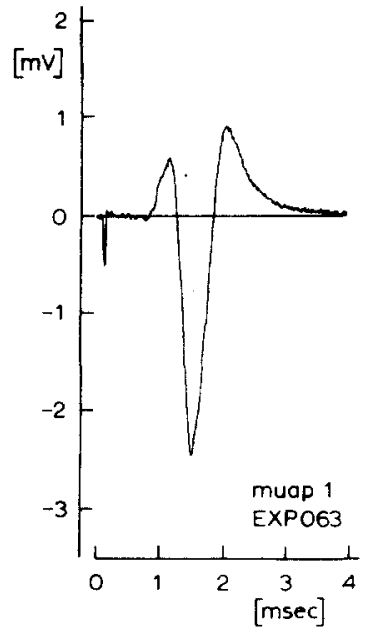

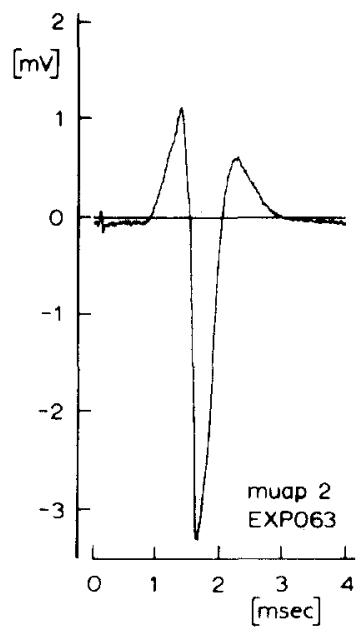
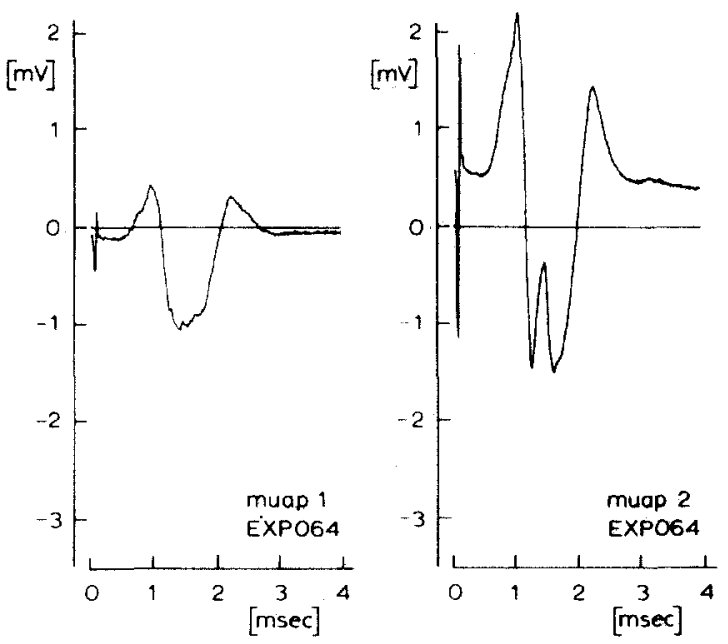

(B)
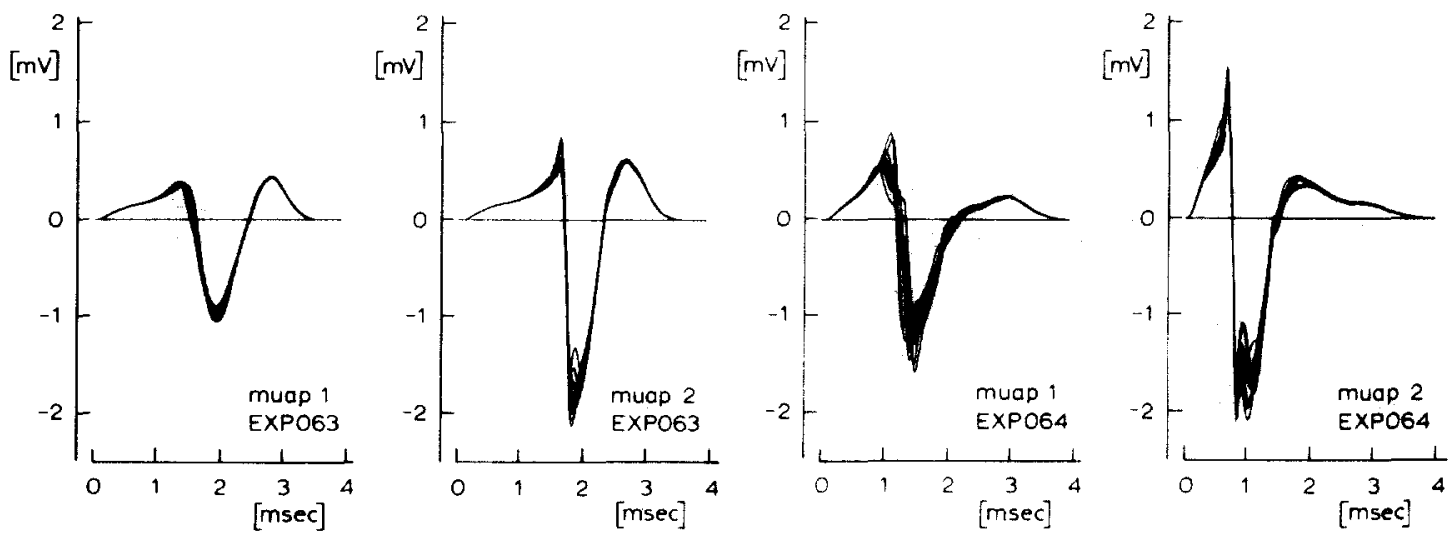

(C)
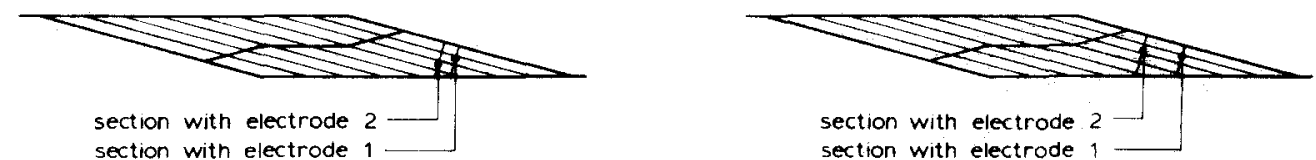

Fig. 7. Comparison of the measured and calculated MUAPs. A: intramuscularly measured MUAPs in exp. 063 and exp. 064. In all responses a stimulus artefact is visible. B: calculated sets of MUAPs (same scale) corresponding to those of A. Each figure shows a set of 10 MUAPs. For MUAP1 all the endplate positions and moments of activation were chosen from probability density functions. For both MUAP2s the endplate position and the moment of activation of the nearest fibre were clamped, $C$ : schematic indication of the muscle sections in which the positions of the electrode and the activated fibres were found. The point indicates the electrode position. 
how this initial and final phase are affected by the parameters describing the onset and the extinction of the single fibre potential.

The dependence of the shape of the calculated MUAPs on the choice of parameters

One of the important questions is of course 'what is the sensitivity of the results to the choice of the parameters?.' In order to examine this question it is good to realize that the parameters used can be divided into three classes. Some parameters have been derived or taken from the literature, some have been measured (as always, with a finite accuracy) and some have been obtained from calcula-
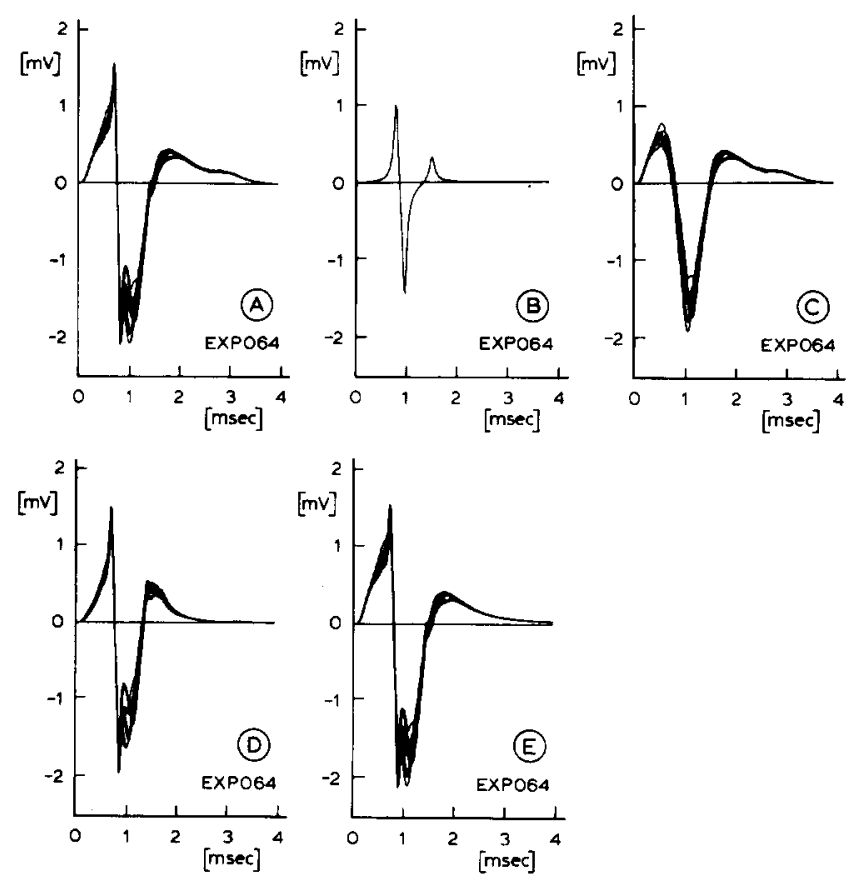

Fig. 8. Dependence of the MUAP on the fibres near the electrode and on the extinction. The calculations concern MUAP2, exp. 064. A: set of MUAPs based on all muscle fibres of the MU (also given in Fig. 7B). $B$ : single fibre potential of the muscle fibre nearest to the electrode. $C$ : set of MUAPs excluding the nearest fibre. D: set of MUAPs of the fibres within a distance of $300 \mu \mathrm{m}$ of the electrode. E: set of MUAPs based on all muscle fibres of the MU, without extinction effects. Note the difference in the second positive phase with respect to the MUAPs shown in A. tions (which means that already a certain 'model' is applied).

One of the disadvantages of a model with a relatively large number of parameters is the practical finding that there is usually more than one combination that results in calculated curves which fit the experimental findings. Therefore a fit is a weak proof of the correctness of the model.

In this section we shall discuss the freedom of choice of the different parameters, together with the effects of this freedom on the calculated results.

The distances $r$ of each activated muscle fibre from the electrode

These parameters have been actually measured and the accuracy of these measurements determine directly the freedom of choice. The accuracy is $\pm 10 \mu \mathrm{m}$. The effect of this accuracy is mainly important with respect to the distance $r$ of the closest fibre. At a distance of approximately $60 \mu \mathrm{m}$ the uncertainty of 10 $\mu \mathrm{m}$ introduces an error of $\pm 20 \%$ in the amplitude of the contribution of this fibre to the MUAP.

In order to investigate the effects of $r$ in a broader sense some calculations were performed. Fig. 8A shows again the set of calculated MUAPs as given in Fig. 7B (exp. 064, MUAP2). Fig. 8B presents the single fibre potential of the nearest fibre $(\mathrm{r}=59 \mu \mathrm{m})$ and in Fig. $8 \mathrm{C}$ the set of MUAPs excluding this nearest fibre is shown. From these results it can be concluded that both the steep positive-negative slope and the split peak of the negative phase were caused by the contribution of the nearest fibre.

In Fig. $8 \mathrm{D}$ the activity of only the fibres within a radius of $300 \mu \mathrm{m}$ around the electrode has been taken into account. This area is commonly considered as the so-called uptake area using a point shaped electrode (Stålberg et al. 1976). According to the model the fibres outside this area add only a low voltage slow amplitude to the MUAP, mainly resulting in an increase in the areas of the negative and last positive phase. 
The position of the endplates and the dispersion in activation time

In a cross-section perpendicular to the muscle fibres the endplates lie in a zone with a thickness of approximately $0.3 \mathrm{~mm}$ (Fig. $6 \mathrm{~A})$. The middle of this zone is represented in the general geometry model by means of 3 straight planes. If the thickness of the motor endplate zone is taken to be twice as large significant changes occur in the calculated MUAPs. The same remark holds for the direction of the endplate 'planes.'

From simulations it can be concluded that the endplate position (in a band with the width used), in combination with the moment of activation, influences considerably the MUAP shape, mainly with respect to the muscle fibres close to the electrode. This means for instance that if the contribution of the single fibre shown in Fig. 8B should occur at any other position on the time axis, the MUAP would have an apparently changed shape.

\section{The tripole parameters}

These parameters have been chosen as a compromise from data derived from the literature. It must be emphasized that the circumstances of the experiments mentioned in the literature differ considerably and, furthermore, some results seem contradictory.

The tripole parameters strongly affect the shape of the single fibre potential and as a consequence also the shape of the MUAP. We feel that the tripole parameters are in fact the 'weakest parameters' of the model. Our compromises on tripole parameter values have not been 'randomly' obtained; of course values have been taken which result in curves with an optimal fit. But, as stated before, this can never be a proof of the correctness of such parameters.

Since the tripole parameters are related to the internal potential $V_{i}(t)$ it would be of great interest to measure simultaneously $V_{i}(t)$ and external potentials at exactly known distances.

\section{Electrical conductivities}

The electrical conductivities have been taken from the rather large range of values. The individual values of $\sigma_{z}, \sigma_{\mathrm{r}}$ and $\sigma_{\text {in }}$ determine the absolute value of the amplitude and the anisotropy $\sigma_{z} / \sigma_{\mathrm{r}}$ affects the shape of the MUAP. From eq. (1) it can be seen that the magnitude is directly proportional to $\sigma_{\text {in }}$. This indicates how the sensitivity of the results is related to this parameter. It must be emphasized that the values of $\sigma_{\mathrm{z}}, \sigma_{\mathrm{r}}$ and $\sigma_{\text {in }}$ finally chosen have not been taken arbitrarily: the chosen values result in reasonable absolute amplitudes. It is worth noting that it was in fact easily possible to obtain reasonable absolute amplitudes with parameter values for the conductivities that lay in the ranges indicated in the literature. At the moment research is being carried out on the electrical conductivities of muscle tissue (Gielen and Boon 1981).

\section{Effects of the extinction of a fibre potential}

All fibres have in fact a limited length and it seems therefore reasonable to study the effects of the extinction.

In Figs. 7B and $8 \mathrm{~A}-\mathrm{D}$ the generation and extinction phases of the action potentials have been taken into account. In Fig. $8 \mathrm{E}$ the influence of the extinction phase is demonstrated: the length of the fibres is infinite, while the endplate positions and activation moments are the same as in Fig. 8A. The comparison between Fig. $8 \mathrm{E}$ and $\mathrm{A}$ shows that the extinction affects to a moderate extent the last positive phase of the MUAP.

\section{Discussion}

The model presented is based on the superposition of the single fibre potentials of the motor unit muscle fibres. This kind of model has been proposed before (e.g., Dimitrova and Dimitrov 1974; Boyd et al. 1978; Griep et al. 1978; Wani and Guha 1980). The essential aspect added in this paper is the direct comparison of the measured and calculated action potentials of motor units of which all the 
muscle fibre positions have been determined. The present results show that it is indeed possible to describe MUAPs to an appreciable degree with our approach.

By labelling the muscle fibres of the MU (glycogen depletion) and by studying the muscle geometry (including the endplate region) the geometry factors have been deduced. If these parameters are substituted in the model, the model is more rigid: there are fewer 'free' parameters and the influence on the results of these remaining free parameters can be studied in more detail.

Besides the group of geometrical parameters 3 other groups can be discussed: the tripole, the electrical conductivity parameters and the parameters determining the activation times. We shall discuss these parameters successively. (Notice that all the calculations and measurements concern action potentials 'found' at electrode positions somewhere between the endplate region and the tendon plate.)

In order to calculate the contribution of a single fibre a tripole model has been used. The choice of the tripole parameters turned out to be of great importance. Boyd et al. (1978), George (1970) and Wani and Guha (1980) used a dipole model, which is certainly not correct: it was impossible to calculate MUAPs, which approximate the recorded MUAP by means of a dipole. When using a dipole the calculated curves for our situation are biphasic, whereas in practice 3 phases are usually observed.

However, the tripole model can be replaced by a still better alternative. After discretization of the intracellular action potential both a solid angle model (Plonsey 1969; Dimitrova and Dimitrov 1974) and a current multipole (Rosenfalck 1969) can be applied; the two approaches are in fact equivalent (Plonsey 1969). These two types of model will certainly result in a more reliable potential contribution of the fibres near the electrode, especially with respect to the second positive phase of the single fibre potential. The shape of this phase is not correct, as can be seen in
Fig. 8B. The introduction of a multipole instead of a tripole is only meaningful if the multipole is related to a well known shape of the internal action potential; this requirement is not yet fulfilled in the case of the EDL muscle.

These considerations led to the conclusion that it is worthwhile to measure the intracellular action potential. This potential provides essential information to reach further insight into MUAP shapes. But we have to consider that special effects may become apparent if the fibres are almost adjacent to the electrode, due to the structure of the packed fibres (Andreassen and Rosenfalck 1978).

Consider, for example, the so-called positive sharp waves (Ruprecht 1974; Ludin 1976). Such rather monophasic potentials can never be obtained with a model having tripole parameters similar to the ones we used. This enforces the statement that these kinds of MUAP model have a limited power if no more evidence on the correctness of the intracellular action potential contribution is given.

The transmembrane current is proportional to the square of the diameter (eq. (1)). This suggests that a more appropriate simulation of the MUAP can be obtained by taking into account every individual diameter. But it is nearly impossible to describe both the 'diameter' of one fibre from its photographically registered shape and its position with respect to the electrode (see Fig. 5). Therefore we used in the MUAP computation one value for the diameters of the muscle fibres of the motor unit. This value was based on the average value of the cross-sectional area of the motor unit fibres (see section on Distance of the activated fibres from the electrode). From simulations it can be concluded that in fact only the diameters of the fibres close to the electrode are of importance.

The contribution of each fibre to the MUAP also depends on the electrical properties of the muscle tissue; with respect to the parameters of the tissue conductivity, it is necessary to determine reliable values. Again these values are lacking in the literature. In 
this field research is going on in our laboratory (Gielen and Boon 1981).

With respect to the parameters determining the activation times it has to be stated that it is nearly impossible to analyse their effects for each muscle fibre in a direct way, as has been done for the geometrical parameters. In addition, the conduction velocity of the muscle fibres will influence the moment at which the fibre action potential arrives at the plane through the electrode perpendicular to the fibre. This last aspect can be quantified in more detail by specific research. But in general the timing aspects will remain uncertain and moreover more or less variable (i.e., jitter). It is evident that the effect of the width of the endplate region on the MUAP is very important. Possible changes during disease have to be considered in clinical EMG practice from this point of view.

Finally we emphasize the general importance of the model for clinical practice. Using the model it is possible to study which parameters can introduce pathological MUAP patterns. Such approaches will become more evidently significant as the model is more reliable. Further research will be directed to refinement of the model, especially with respect to the intracellular action potential(s) and the electrical conductivities. Moreover, more attention will be paid to the link between the model and pathological processes (Boon and Pelsmaeker 1981).

\section{Summary}

In order to increase insight into the electrical phenomena of active motor units, a computer simulation model has been developed. With this model motor unit action potentials (MUAPs) have been calculated.

The model has been based on the superposition of the muscle fibre potentials of the fibres of one motor unit. For verification, calculated MUAPs have been compared with the matching recorded MUAPs.

During experiments one motor unit was stimulated and the MUAP of this unit was measured with intramuscular wire electrodes. After the experiments the positions of the activated fibres of this unit and of the electrodes were determined by means of histochemical techniques. Other parameters were derived from other experiments or the literature. Using the obtained set of parameters in the model MUAPs were calculated. These MUAPs were compared with the measured MUAPs.

From this comparison it has been concluded that the model predicts the MUAP to an appreciable degree.

The results clearly show the dominating effect of muscle fibres in close vicinity of the electrode and the important effect of the activation moment of those fibres on the shape of the MUAP.

\section{Résumé}

Calcul et enregistrement du potentiel d'action de la même unité motrice

On a mis au point un modèle de simulation par ordinateur en vue d'élucider les phénomènes électriques dans les unités motrices actives. Des potentiels d'action d'unités motrices (MUAP) ont été établis avec ce modèle, qui est basé sur la superposition des potentiels des fibres d'une unité motrice. Comme vérification, les MUAP calculés ont été comparés aux les MUAP correspondants enregistrés.

En cours d'expérience, on a stimulé une unité motrice et recueilli son MUAP au moyen d'électrodes-fils intramusculaires. Après les expériences on a déterminé la position des fibres activées et celle des électrodes à l'aide de techniques histochimiques. On a également utilisé des paramètres déduits d'autres expériences, ou de la bibliographie, et calculé les MUAP dans le modèle en se servant de cet ensemble de paramètres. Ces MUAP ont été comparés aux MUAP mesurés: on en a conclu que le modèle pouvait correctement prévoir le potentiel d'action musculaire unitaire. 
Ces résultats montrent clairement l'effet dominant des fibres musculaires qui sont au voisinage immédiat de l'électrode, et l'importance de leur instant d'activation, sur la forme du MUAP.

The authors are indebted to Ton Verloop for valuable technical help and to Truus Steijlen for secretarial assistance.

\section{References}

Andreassen, S. and Rosenfalck, A. Recording from a single motor unit during strong effort. IEEE Trans. biomed. Engng, 1978, 25: 501-508.

Baumgarten, R. von, Kanzow, E., Koepchen, H.P. und Timm, F. Beitrag zur Technik der extra- und intracellulären, sowie der stereotaktischen Mikroableitung im Gehirn. Pflügers Arch. ges. Physiol., 1960, 271:245-256.

Boon, K.L. and Pelsmaeker, A.R.Th. On-line analysis of motor unit action potentials and the interpretation of the parameters. Contribution Strathclyde Bioengineering Seminar on Computing in Medicine, Glasgow, 1981. Macmillan Publishers, Basingstoke, in press.

Boyd, D.C., Lawrence, P.D. and Bratty, P.J.A. On modeling the single motor unit action potential. IEEE Trans. biomed. Engng, 1978, 25: 236-243.

Buchthal, F., Guld, C. and Rosenfalck, P. Multielectrode study of the territory of a motor unit. Acta physiol. scand., 1957, 39: 83-104.

Clark, J.W, and Plonsey, R. A mathematical evaluation of the core conductor model. Biophys. J., 1966, 6: 95-112.

Close, R. Dynamic properties of fast and slow skeletal muscles of the rat during development. J. Physiol. (Lond.), 1964, 173: 74-95.

Coërs, C. and Woolf, A.L. The Innervation of Muscle. Blackwell, Oxford, 1957.

Dimitrov, G.V. Computed extracellular potentials of a number of muscle fibres. I. communication. Influence of the desynchronization in the fibres activation. EMG clin. Neurophysiol., 1978a, 18: $361-376$

Dimitrov, G.V. Computed extracellular potentials of a number of muscle fibres. II. communication. Effects of axial displacement of the muscle fibres. EMG clin. Neurophysiol., 1978b, 18: 517-526.

Dimitrova, N. Model of the extracellular potential field of a single striated muscle fibre. EMG clin. Neurophysiol., 1974, 11: 53-66.

Dimitrova, N. and Dimitrov, G.V. Extracellular potential field of a single striated muscle fibre. EMG clin. Neurophysiol., 1974, 14: 279-292.

Ekstedt, J. Human single muscle fiber action potentials. Extracellular recording during voluntary and chemical activation. With some comments on endplate physiology and on the fiber arrangement of the motor unit. Acta physiol. scand., 1964, 61 (suppl. 226): 16-20.

Fatt, $P$. An analysis of the transverse electrical impedance of striated muscle. Proc. roy. Soc. B, 1964, 159B: $606-651$.

Geddes, L.A. and Baker, L.E. The specific resistance of biological material. A compendium of data for the biomedical engineer and physiologist. Med. Biol. Engng, 1967, 5: 271-293.

George, R.E. The summation of muscle fibre action potential. Med. Biol. Engng, 1970, 8: 357-365.

Gielen, F.L.H. and Boon, K.L. Measurements on the (complex) electrical conductivity and the anisotropy in skeletal muscle; a new electrode configuration. In: Vth Int. Conf. Electr. Bio-Impedance. Business Center for Academic Societies, Tokyo, 1981: 191-194.

Griep, P.A.M., Boon, K.L. and Stegeman, D.F. A study of the motor unit action potential by means of computer simulation. Biol. Cybernet., 1978, 30: 221-230.

Griep, P.A.M., Pool, Ch.W. and Boon, K.L. Modeling (and verification) of the motor unit action potential taking into account finite muscle fibre length. In: 4th Int. Congr. Electrophysiol. Kinesiology, Boston, Mass., 1979: 192-193.

Griep, P.A.M., Pool, C.W., Lammerée, G.C., WallingaDe Jonge, W., Seeder, T. and Donselaar, Y.E. Intramuscular and epimuscular microstimulation of single motor units. Neurosci. Lett., 1980, 17: 191-196.

Hanson, J. The effects of repetitive stimulation on the action potential and the twitch of rat muscle. Acta physiol. scand., 1974, 90: 387-400.

Hopf, H.C. and Struppler, A. Elektromyographie, Lehrbuch und Atlas. Georg Thieme, Stuttgart, 1974: 229 pp.

Hubbard, J.I. and Schmidt, R.F. Electrophysiological investigation of mammalian motor nerve terminals. J. Physiol. (Lond.), 1963, 166: 145-167.

Kugelberg, E. Properties of the rat hind-limb motor units. In: J.E. Desmedt (Ed.), New Developments in EMG and Clinical Neurophysiology, Vol. 1. Karger, Basel, 1973: 2-13.

Lindström, L. and Broman, H. A Model Describing the Power Spectrum of Myoelectric Signals. II. Motor Unit Signal. Techn. Rep. 8.74. Chalmers University, Göteborg, 1974.

Lorente de Nó, R. A study of nerve physiology. Stud. Rockefeller Inst. medical Res., 1947, 132: 384477.

Ludin, H.P. Praktische Elektromyographie. Ferdinand 
Enke Verlag, Stuttgart, 1976.

Muscholl, E. Elektrophysiologische Untersuchung der einzelnen Faseranteile des isolierten Rattenzwerchfelles. Pflügers Arch. ges. Physiol., 1957, 264: $467-483$.

Plonsey, R. Bioelectric Phenomena. McGraw-Hill, New York, 1969.

Plonsey, $R$. The active fiber in a volume conductor. IEEE Trans. biomed. Engng, 1974, 21: 371-381.

Pool, C.W., Donselaar, Y.E. and Griep, P.A.M., The $\alpha$-glucan-uridine diphosphate glycosyltransferase reaction for the identification of glycogendepleted muscle fibers. J. Histochem. Cytochem., 1978, 26: 742-744.

Rosenfalck, P. Intra- and Extracellular Potential Fields of Active Nerve and Muscle Fibres. A Physico-mathematical Analysis of Different Models. Akademisk Forlag, Copenhagen, 1969.

Ruprecht, E.O. Befunde bei Neuropathien. In: H.C.
Hopf and A. Struppler (Eds.), Elektromyographie, Lehrbuch und Atlas. George Thieme Verlag, Stuttgart, 1974: 37-65.

Rush, S., Abildskov, J.A. and McFee, R. Resistivity of body tissues at low frequencies. Circulat. Res., 1963, 12: 40-50.

Stålberg, E. and Trontelj, J.V. Single Fibre Electromyography. Mirvalle Press, Woking, 1979.

Stålberg, E., Schwartz, M.S., Thiele, B. and Schiller, H.H. The normal motor unit in man. A single fibre EMG multielectrode investigation. J. neurol. Sci., 1976, $27: 291-301$.

Wani, A.M. and Guha, S.K. Synthesising of a motor unit potential based on the sequential firing of muscle fibres. Med. Biol. Engng Comput., 1980, 18: 719-726.

Yonemura, K. Resting and action potentials in red and white muscles of the rat. Jap. J. Physiol., 1967, 17: 708-719. 